\title{
DESIGN AND DEVELOPMENT OF TWO WHEELER TO AVOID TRIBLE RIDERS AND ACCIDENTS
}

\author{
R. VENKATAKRISHNAN ${ }^{1}$, S. JAYALAKSHMY ${ }^{2}$, S. JAGADEESAN ${ }^{3}$, LR. DURAIPANDI $^{4} \&$ S. $^{2}$ \\ SAISUMANTH ${ }^{5}$ \\ ${ }^{1,2}$ Assistant Professor, Department of Mechanical Engineering, IFET College of Engineering, Villupuram, Tamil Nadu, India \\ ${ }^{3,4,5}$ UG Student, Department of Mechanical engineering, IFET College of Engineering, Villupuram, Tamil Nadu, India
}

\begin{abstract}
Road site visitor's accidents (RTA) have emerged as an essential public health problem which needs to be tackled by a multi- disciplinary approach. The vogue in RTA accidents and dying is turning into alarming in international locations like in India. The strategy to put in force the regulations and law on hand to prevent road accidents is regularly ineffective. The accidents in most cases happens due to extra then two riders and now not sporting security helmets in bike, which can be averted by means of making a bike in smart way the use of smart devices. We grant a bike consist of helmet detector built in in bike, piezoelectric sensor, Load phone device, in addition with electricity generation gadget. Load mobile system and piezoelectric sensor are used to realize the number of rides on the two wheeler which can help to avoid triple riders. Helmet detector is to recognize those folks who put on helmet or now not by means of using raspberry pi 3 By these clever bike accidents will be reduced. In addition Power generation made through TEC which working the precept of peltier effect.

KEYWORDS: Smart Bike, Bike Inbuilt Helmate Detector, Avoiding Triple Riders, Power Generation System Replacing Dynamometer
\end{abstract}

Received: Jun 08, 2020; Accepted: Jun 28, 2020; Published: Sep 09, 2020; Paper Id.: IJMPERDJUN20201128

\section{INTRODUCTION}

Mostly the usage of 4 wheelers is extensively decreased than the two wheelers. Which means usage of two wheelers by way of the society had extended more? Riding on two wheelers with over load, more than two members on bike and not wearing the helmet humans are regularly located on India. This a essential trouble that leads to unexpected and tremendous accidents. To reduce the probability of accident the format and implementation of clever bikes with clever units have been preceded.

The World Health Organization (WHO) reports, India is susceptible for street accidents and most of the instances are of two wheeler accidents. From the latest surveys in India 4 individuals are loss of life for each and every one hour due to no longer wearing helmet and additionally many accidents are taking place due to more than 2 riders on bike. On the basis of these troubles we added a device which is to observe the helmet and those riders extra than two on bike.

Avoiding triple in bike is the use of system is such as strain sensor, relay, Arduino. The strain sensor used to recognize the strain utilized on tank due to more than two riders on it and it sends the strain value to Arduino then there is threshold restriction for that strain in Arduino programming based totally on that restriction it will become aware of if there are more than two riders on bike an it will off the engine ignition if extra than two riders 
on the bike the use of relay.

Helmet detector to locate whether or not the man or woman wearied the helmet or not. The components for this notion are night version camera, raspberry pi. The digital camera image sends to the raspberry pi and will procedure the photograph and using tensor drift we can notice whether the individual wearer the helmet or not. The every other notion that is applied in bike for strength technology using the machine such as TEC, air cooler(fins),stainless steel.TEC is carried out with the peltier effect. The TEC is to produce the electricity the usage of thermal electricity from the bike. It works based on temperature change. It have two facets one is warm and any other one is cool side.

The hot aspect attaches to the silencer and the cool facet attach with fins from the alternate in temperature strength will be generate.

\section{DESCRIPTIONS}

\section{Existing System}

Traffic police is only the solution having proper comprehend in the Society. Over the years, there have been quite a few regulations made by way of the Government to manipulate those riders who are not wearing helmet and using greater than two people bike. The a variety of acts for these things to do in India are The visitors nice for using a two-wheeler except wearing a helmet is Rs.1,000, up from historical penalty of Rs.100. Not carrying helmets whilst riding two-wheelers on roads in Madhya Pradesh are automatically challenged below Section 129 of the Motor Vehicles (MV) Act, 1988, which makes carrying of the protective headgear mandatory. There is also gathering fines when extra than two riders on bike.

\section{Drawbacks of Existing System}

- $\quad$ Bribe

- Not following generally

- Faultysystem

- Influence and partiality

The proposed system helps to avoid these drawbacks.

\section{PROPOSED SYSTEM AND ITS EXPLANATION}

The smart bike consist of in most cases three standards have been involved. They are helmeted detector built in bike, fending off three riders in bike, energy generation changing via dynamometer, increasing efficiency of engine

\section{Helmet Detector}

The raspberry pi night time model camera which is to capture the riding individual in the bike. The raspberry pi digital camera at once connected with the raspberry pi. The raspberry pi analyze the captured photographs from the camera, then the use of the tensor drift in raspberry pi can notice the rider of bike is wearer helmet or now not if the rider not put on the helmet then the sign ship to the relay which cutoff the ignition of engine.

The Raspberry Pi is a savings card-sized single-board computer. Raspberry Pi has a Broadcom BCM2835 system on chip (SoC), which consists of an ARM1176JZFS $700 \mathrm{MHz}$ processor, Video Core IV GPU, and used to be originally 
shipped with 256 megabytes of RAM, later upgraded (Model B \& Model B+) to 512 MB. Pi two Model B runs 6X Faster than the $\mathrm{B}+$, and comes with $1 \mathrm{~GB}$ of RAM--that's double the amount of RAM of the preceding model.

The digital camera module utilizes the devoted CSi interface, which is placed behind the Ethernet port on the Raspberry Pi .The Raspberry Pi Camera Module as proven in the Figure 3.6 is a 5MP CMOS digicam with a fixed focus lens that is successful of capturing still images as nicely as excessive definition video. Stills are captured at a resolution of $2592 \times 1944$, while video is supported at 1080p at 30 FPS, 720p at 60 FPS and 640x480 at 60 or 90 FPS. . This makes it perfect for projects such as hidden security cameras, excessive altitude balloon experiments, and even an onboard dig cam for RC automobile adventures. The digicam is supported in the ultra-modern version of Raspbian, Raspberry Pi's desired operating system.

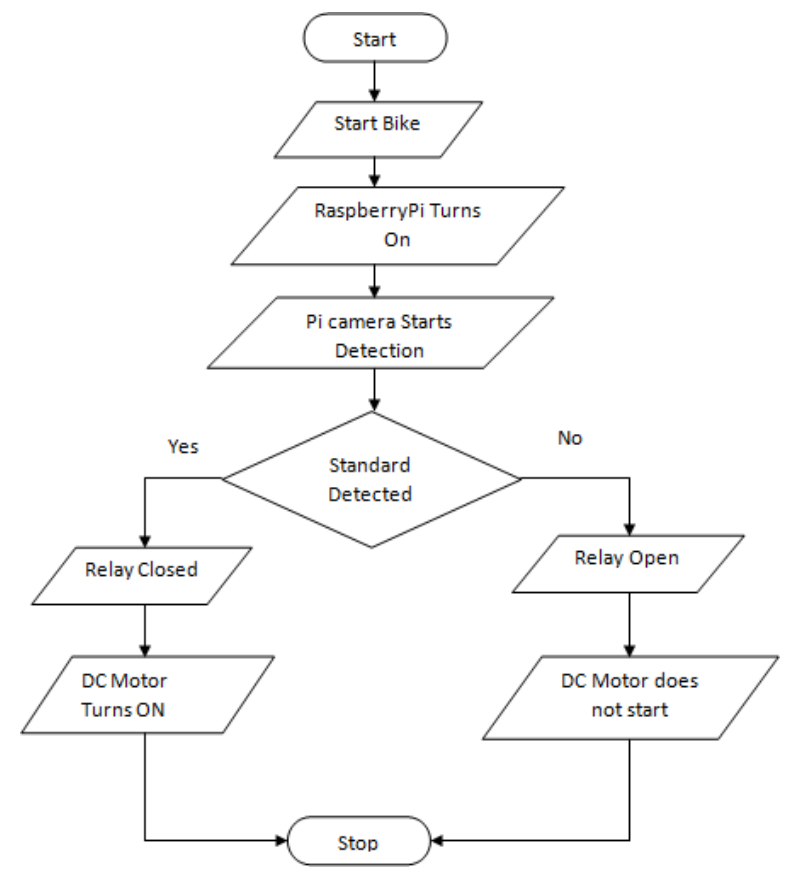

Figure 1: Flow Chart for Hellmate Detector System

\section{Triple Riding Avoider}

In this module there is a strain sensor which is placed at the aspect of the tank of bike due to the fact at that spot only more strain will be there if more than two riders are there on the bike. This strain sensor sends the sensed strain value to the Arduino then it compares with threshold price which is programmed in it and if the price is greater than the threshold price then it prompts relay and offs engine ignition.

A piezoelectric sensor is a device that uses the piezoelectric effect to measure changes in pressure, acceleration, temperature, strain, or force by converting them to an electrical charge. The high modulus of elasticity of many piezoelectric materials is comparable to that of many metals and goes up to $106 \mathrm{~N} / \mathrm{m}^{2}$.

\section{Power Generation Using Tec}

In TEC thermoelectric materials are packed between the hot side and the cold-side warmness exchangers. The thermoelectric materials are made up of p-type and n-type semiconductors, whilst the warmness exchangers are steel plates with high thermal conductivity. 
Table 1: Piezoelectric Thresholds

\begin{tabular}{|c|c|c|c|}
\hline Principle & 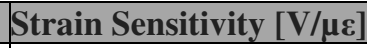 & Threshold $[\mu \varepsilon]$ & Span to Threshold Ratio \\
\hline Piezoelectric & 5.0 & 0.00001 & $100,000,000$ \\
\hline Piezo-resistive & 0.0001 & 0.0001 & $2,500,000$ \\
\hline Inductive & 0.001 & 0.0005 & $2,000,000$ \\
\hline Capacitive & 0.005 & 0.0001 & 750,000 \\
\hline Resistive & 0.000005 & 0.01 & 50,000 \\
\hline
\end{tabular}

The temperature distinction between the two surfaces of the thermoelectric module generates electricity using the Peltier Effect. When thermal electricity releases from the silencer TEC hot facet part observes it and from the fins it get cool air via the mixture of this two warm and cool components there will be a temperature change, from this temperature trade electricity will be generated.

Thermoelectric generator modules are strong gadget which can convert warmness or temperature difference into electrical energy. Thermoelectric factors are made of P-type and N-type semiconductor.

Thermoelectric modules are primarily based on Peltier effect. When there is a temperature difference between two aspects of semiconductor, a voltage is created. Current flows from $\mathrm{N}$ kind factor and passes into $\mathrm{P}$ type element.

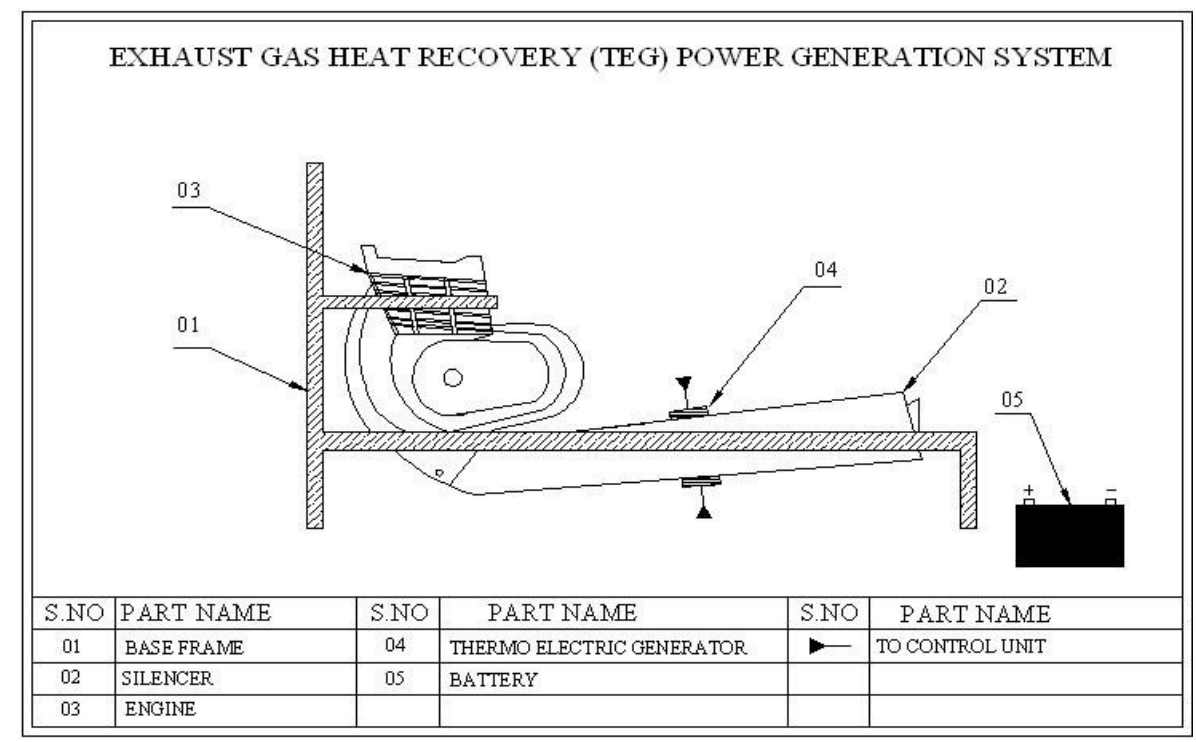

Figure 2: Power Generation System Using Tec.

Thermoelectric modules are devices that either convert thermal strength from a temperature gradient into electric energy or vice versa, convert applied electric power into a temperature gradient.

Table 2: TEG Materials

\begin{tabular}{|c|l|c|}
\hline S. NO. & \multicolumn{1}{|c|}{ TEG MATERIAL } & TEMPERAT URE RANGE \\
\hline 1 & $\begin{array}{l}\text { Alloys based on Bismuth } \\
\text { (Bi) in combinations with } \\
\text { Antimony(An), Tellurium } \\
\text { (Te) or Selenium (Se) }\end{array}$ & Low temperature up to 450K \\
\hline 2 & $\begin{array}{l}\text { Materials based on alloys } \\
\text { of Lead (Pb) }\end{array}$ & Intermediate temperature up to 850K \\
\hline 3 & $\begin{array}{l}\text { Material based on Si- } \\
\text { Ge alloys }\end{array}$ & Higher temperature up \\
\hline
\end{tabular}




\section{ADVANTAGES OF PROPOSED SYSTEM}

- Directly implement in vehicle

- Improves safety of rider

- Improves mechanical efficiency due to TEC

\section{CONCLUSIONS}

This smart bike is established with the newly progressive three concepts, they are inbuilt helmet detector in bike, heading off triple riders in two wheelers, energy era system. in accordance to power era system, The electrical power technology of the thermoelectric generator is observed to be a strong feature of glide charge and inlet exhaust temperature. The temperature difference between the warm and cold junctions of TEG accelerated as the engine speed or the coolant temperature increase. The output voltage, in accordance to the Peltier effect, additionally improved as the temperature difference increase. Therefore, the output energy and thermal efficiency can be improved. The parametric contrast of the longitudinal model indicates that TEG performance improves for configurations that have minimum TEG peak and most TEG width. The high-efficiency warmness exchanger is quintessential to enlarge the amount of warmness strength extracted from the exhaust gas. It is located that exhaust gasoline parameters and warmness exchanger structure have a big effect on the system power output and the strain drop. The find out about additionally recognized the potentials of the technologies when included with other devices to maximize the potential strength efficiency of the vehicles. In warding off triple riders thought the triple using can keep away from by piezo electric detection and relay. The bike inbuilt helmet detector which assist to compulsory wear of helmet whilst riding. By implementing this clever bike clearly 50\% of accidents are prevented. Because the recent survey shows that $90 \%$ of avenue accidents, dying and damages are happens due to no longer sporting helmet and triple riders in the bike. Hence most accidents and addition of energy era is made by way of this clever bike.

\section{REFERENCES}

1. Baleswar Kumar Singh, Dr.Shrivastava. "Exhaust Gas Heat Recovery For I.C. Engine-A Review”, Int. Journal Of Science And Research Technology,2014.

2. Prathmeshramade, Manojshelar, "Automobile Exhaust thermoelectric Generator Design And Performance Analysis", Int. Journal Of Emerging Technology and Advanced Engineering,2014.

3. Vasanthi Ajay \& Soumya Manjunath Chavan, "Stereotype and Culture in Indian Advertisements “, BEST: International Journal of Humanities, Arts, Medicine and Sciences (BEST: IJHAMS), Vol. 4, Issue 2,pp. 61-64

4. $\quad$ R K Rajput, "Heat And Mass Transfer”, Third Edition, Pub.-Tata Mcgraw-Hill (2009)

5. Chen L, Li J, Sun F, Wu C. Performance Optimization Of A Two Stage Semiconductor Thermoelectric Generator. Appl Energy 2005; 82:300- 312. 6. Www. Wikipidia.Org.

6. Darren Quick's Thermoelectric Report.

7. Vasanthi Ajay \& Soumya Manjunath Chavan, "Stereotype and Culture in Indian Advertisements", BEST: International Journal of Humanities, Arts, Medicine and Sciences (BEST: IJHAMS), Vol. 4, Issue 2, pp. 61-64

8. Http://Timesofindia.Indiatimes.Com/Toir Eporter/Author-Dipak-K-Dash-479213512.Cms 
9. Http://Timesofindia.Indiatimes.Com/Toir Eporter/Author-Dipak-K-Dash-10519.Cms

10. Rattapoomwaranusast, Nannaphatbundon, Vasantimtong And Chainarontangnoitechniques For Motorcycle Safety Helmet Detection," 2013,28th International Conference On Image And Vision Computing New Zealand.

11. Romuere Silva, Kelson Aires, Thiago Santos, Kalyf Abdala, Rodrigo Veras "Atomatic Detection of Motorcyclists Without Helmet, "Departamento De Computacouniversidade Federal Do Piaui Teresina,Brazil.

12. Upasana Saxena, "Uberification: A Disruptive Innovation in Ridesharing Revolution 3", IMPACT: International Journal of Research in Business Management (IMPACT: IJRBM), Vol. 7, Issue 7, pp. 1-12

13. Ping Li, Ramymeziane, Martin J,Hassanezzaidi,Philippecardou, "A Smart Safety Helmet Using Imu And Eeg Sensors For Worker Fatigue Detection," Reparti Center, Laval University

14. Quebec, Canada.

15. Manjesh N, Prof. Sudarshan Raj,”Smart Helmet Using Gsm \&Gps Technology For Accident Detection And Reporting System," International Journal Of Electrical And Electronics Research Issn 2348-6988 (Online) Vol. 2, Issue4.

16. Vitthal V Parab, “Glimpses of Modern India in Gita Mehta's 'Snakes and Ladders' “, BEST: International Journal of Humanities, Arts, Medicine and Sciences (BEST: IJHAMS), Vol. 3, Issue 7, pp. 59-68

17. Mohd Khairul Afiqmohdrasli, Nina Korlinamadzhi, Juliana Johari , "Smart Helmet Sensors For Accident Prevention,” 2013 International Conference On Electrical Electronics And System engineering. 